I. INSTABILITY IN DWARF STARS OF

LATER TYPE: T TAURI STARS AND

RELATED OBJEGTS, AND THE

UV CETI VARIABLES 


\title{
1. ON THE NATURE AND ORIGIN OF THE T TAURI STARS
}

\author{
GEORGE H. HERBIG \\ Lick Observatory, Mount Hamilton, California, U.S.A.
}

It is fitting that, in opening this Symposium with a consideration of the $\mathrm{T}$ Tauri stars, we note that this month marks the tenth anniversary of the September 1945 issue of the Astrophysical Fournal, in which appeared the remarkable pioneering paper by Alfred $\mathrm{H}$. Joy that initiated the study of emission-line stars associated with nebulosity. This contribution opened a new approach to the study of the relationship of stars to their environment. Today, ten years later, the $\mathrm{T}$ Tauri stars and their interaction with nebular material form a topic whose significance we may not fully appreciate and whose opportunities have been as yet only superficially exploited.

In the decade that has passed since the appearance of Joy's I945 paper, a great deal of work has been done in this field (most of it, I should remark, by astronomers who are to participate in this Symposium). Rather than attempting to summarize these ten years of progress, I wish to do only two things: first, to present an assessment of the problem of the nature of the $\mathrm{T}$ Tauri stars as it stands today, and second, to discuss some evidence that may bear on the question of the origin of these objects. If a satisfactory solution to even one of these problems can be found, it will then be possible to look for a deeper significance in the instability of the $\mathrm{T}$ Tauri stars.

For those who are not familiar with the subject, it should be said that the $\mathrm{T}$ Tauri stars are low luminosity objects having a rather characteristic emission-line spectrum, are irregularly variable in light and spectrum, and have been found only in association with nebular material, both bright and dark. The observational data are now so extensive that the reality of the association with nebulae is quite beyond dispute. The central question is: are the $\mathrm{T}$ Tauri variables new stars, recently formed or still forming within the nebulae, or are they ordinary field stars that have encountered the gas and dust clouds accidentally, and are in process of being modifiedperhaps rather superficially-by their environment?

Three years ago [1], in an examination of the information then available, I concluded that it was 'not possible to make a clear-cut and entirely acceptable decision between [these] two opposing alternatives on the basis 
of observational evidence alone'. Today, in my opinion, we are able to take a more positive stand. I believe that the evidence now available favours the hypothesis that the $\mathrm{T}$ Tauri stars as a class are new objects, genetically associated with the clouds in which they are found. Presumably their instability is a consequence of their relative youth. Some of the evidence to this effect is still ambiguous, but $I$ believe that if one were to maintain the opposite position, and try to explain away all the observational facts by ad hoc processes involving normal stars, he would find himself in an unrealistic position.

First consider three observational facts that must be mentioned here because of their undoubted significance, but which in my opinion do not by themselves enable us to reach a firm decision.

(I) It has been found [1] that the T Tauri stars have somewhat diffuse absorption lines, a characteristic which is known to be quite abnormal for ordinary single stars of the same spectral types [2]. Although only the brightest $\mathrm{T}$ Tauri stars are accessible to observations of this kind, there is indication that other stars of the group also may possess this peculiarity.

(2) The T Tauri stars of later type are found to be systematically brighter than main sequence stars of the same spectral types [1]. Some of the M-type objects in the Taurus clouds, for example, lie 2 to 3 magnitudes above the main sequence even before any allowance is made for absorption. Similar departures from the main sequence have been found in NGG 2264 [3].

(3) If the $T$ Tauri stars are field stars in a stage of active accretion, one might expect that their spectra would give some evidence of the infall of material. However, there is no clear spectroscopic evidence that matter is actually being collected by the $T$ Tauri stars. In fact, the conclusion which emerges from both low-[4] and high-dispersion [5] spectroscopic observations is that the emission-line regions of their atmospheres, as well as certain higher layers that produce a shell-type spectrum, are rising with respect to the source of the underlying absorption spectra. Since the absorption spectra presumably indicate the radial motion of the stars, one would expect to observe just such an effect if the $\mathrm{T}$ Tauri stars were ejecting material, rather than collecting it.

These three characteristics - line width due to rapid rotation or largescale turbulence, abnormal luminosity, and lack of evidence for the infall of matter-neither demonstrate nor disprove that the $\mathrm{T}$ Tauri stars are newly-formed objects. It is true that newly-formed stars might exhibit abnormally high rotation as a result of the conservation of angular momentum during formation, and that the ejection of material would be a way of disposing of excess momentum. It is also true that the abnormally 
bright $\mathrm{T}$ Tauri stars inhabit a portion of the spectrum-luminosity diagram where lie the evolutionary tracks of gravitationally contracting stars having a mass somewhat less than the sun's [6, 7]. But as interesting as it would be to identify the phenomena observed in the T Tauri stars with these theoretical concepts, one cannot use such correspondences to prove the correctness of the basic assumption. It might be that the collection of a great deal of material by a normal star could somehow give rise to these same effects. Fortunately, there are three other points which bear on this problem that cannot be evaded so readily.

(4) The data that are now available on the occurrence of emission-line stars in the Taurus dark clouds $[4,8,9]$ make it possible to compute, under reasonable assumptions, the average number of such stars per cubic parsec of these nebulae. In the central region of the Taurus clouds, it is found that there are, depending upon the initial assumptions, from 5 to 15 times as many $\mathrm{T}$ Tauri stars per unit volume, down to about apparent visual magnitude 17.5 , as there are stars in the same absolute magnitude interval in the region of the sun. Allowance for undetected T Tauri-like variable stars which (temporarily or permanently) show no emission lines might increase these figures from 50 to $100 \%$. In other words, there are far more T Tauri stars in these clouds-by one order of magnitude-than can be accounted for by the random encounter of field stars with the clouds.

(5) In some ncoulae, there have been found curious concentrations of emission-line objects in the vicinity of a much brighter early-type star imbedded in the cloud. In this small region, the star density may become rather large. The most obvious explanation of such a concentration is that, for some reason, stars are actually being formed there, since field stars would be scattered at random through the nebula. Whether the presence of the early-type star is the cause, or is a consequence of the favourable conditions for star formation prevailing in that area is an open question.

If, then, the $\mathrm{T}$ Tauri stars are newly-formed objects, it might be asked if there is any observational indication as to how they begin their careers. This is a dangerous question to attempt to answer at the present time because of the considerable variety of objects that are found in association with nebulae. Our lack of any general understanding of the processes which are taking place may well cause one to attach undue importance to phenomena which are, in reality, not of major significance. Furthermore, one must refrain from generalizing too far on the basis of limited information, because it is entirely possible that the $T$ Tauri stars can originate in more than one way. With these reservations, let us consider some observational results which appear to bear upon this question. 

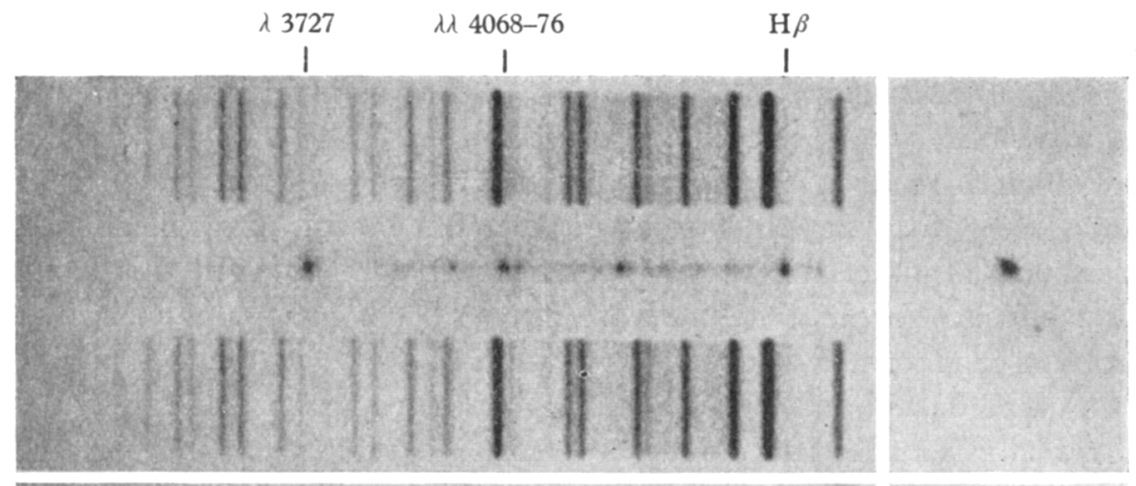

Haro $11 a$

Herbig 1
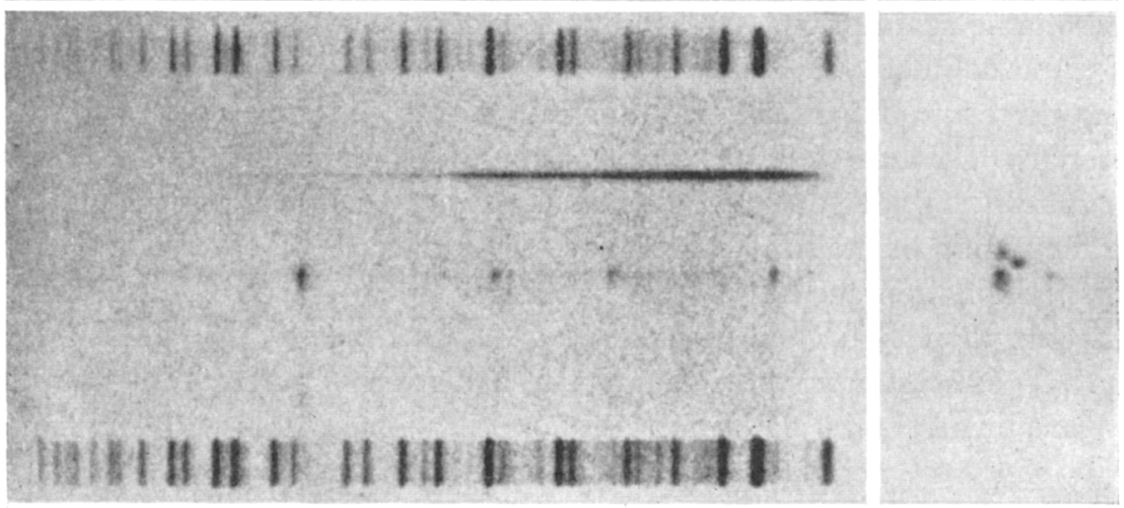

Haro $12 a$

Herbig 2
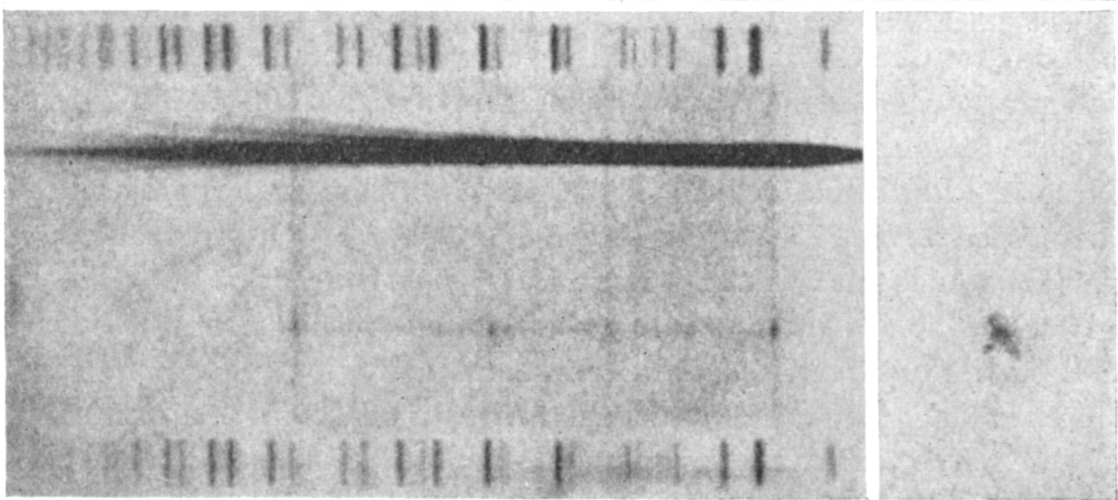

Haro $14 a$

Fig. I. Slit spectrograms and direct photographs of three Herbig-Haro Objects in Orion. The dispersion of the original spectrograms is $430 \AA / \mathrm{mm}$. at $\mathrm{H} \gamma$; they were obtained with the nebular spectrograph of the Crossley reflector of the Lick Observatory. The direct photographs, which do not match the spectrograms either in scale or in orientation, were obtained with the same telescope and with blue-sensitive plates. In all the direct photographs, north is at the top and east to the right. The spectrogram of Haro $12 a$ refers to the knot of stars and nebulosity at the lower (southern) edge of the Object. 
In a dense dark cloud not far from the Orion Nebula lie the brightest known examples of a very peculiar class of nebulous stars that have been called the 'Objects of Group D' by Herbig [1], and the 'Herbig-Haro Objects' by V. Ambartsumian [10]. (I hope that I shall be forgiven if I employ here the latter designation, which seems less clumsy than the other.) They have been studied by Herbig [11], Haro [12], and, very recently, by K.-H. Böhm [13]. Very briefly, the simplest of these Objects consist of a stellar or semi-stellar nucleus together with a very small emission nebula, whose strongest lines beside the Balmer series are those of [O I], [O II], and $[\mathrm{S} \mathrm{II}]$, which occur with rather unusual relative intensities (see Fig. I).

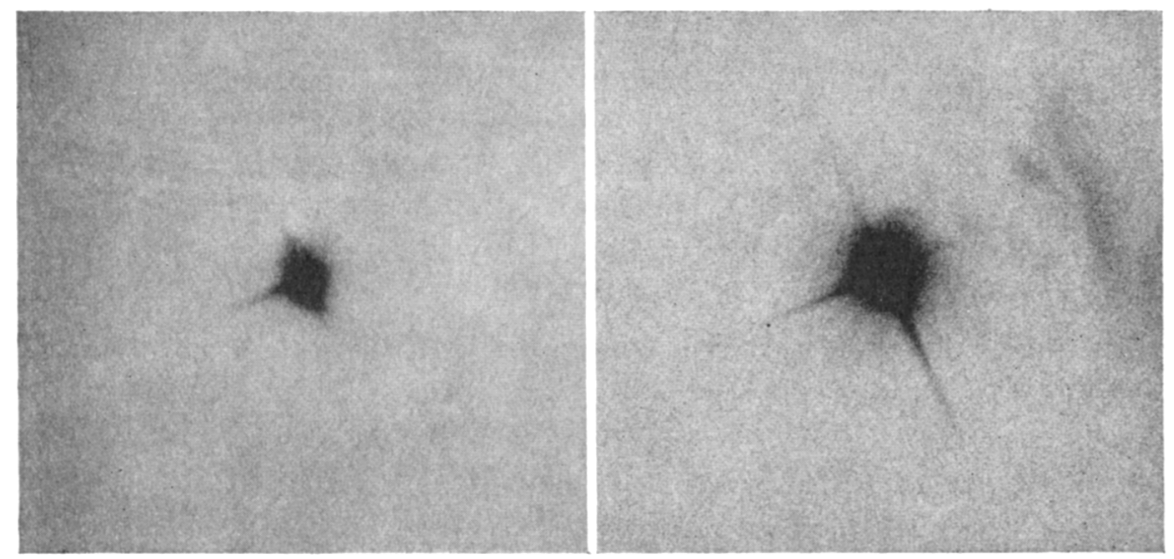

Fig. 2. The nebulosity immediately surrounding $T$ Tauri. Both photographs were obtained with the Ioo-inch Mount Wilson reflector by Dr W. Baade, to whom I am indebted for the privilege of reproducing them here. North is below and east to the left. The left-hand photograph is a short exposure obtained on 19 December 1944, and the right-hand is a longer exposure taken on I October 1940. The latter photograph shows, on the extreme right, the brighter portion of NGC 1 555, Hind's Variable Nebula. The elongation of the image of T Tauri by bright nebulosity in position angles $151^{\circ}$ and about $33^{\circ}$, discovered visually by Burnham, is evident, as is a nebulous flare in about $190^{\circ}$. The sharp bright rays in p.a. $60^{\circ}, 150^{\circ}$, etc. . . are due to the supports of the Newtonian flat mirror of the telescope.

Accompanying this nebular spectrum, at least in the brightest Objects, is a weak, low-temperature continuum and a set of bright lines due to easily produced ions, such as $\mathrm{Ca}$ II and [Fe II]. This second spectrum, although heavily masked by the nebular emission lines, is reminiscent of the spectra of some $\mathrm{T}$ Tauri stars.

Consequently, it is a fact of extraordinary interest that $\mathrm{T}$ Tauri itself is surrounded by a very small nebula [14] (see Fig. 2) whose bright-line spectrum is very similar to those of the Herbig-Haro nebulae. If the source of the $\mathrm{T}$ Tauri-like spectrum in the brightest Herbig-Haro Objects- 
presumably a star-should simply brighten up by 4 or 5 magnitudes, then the result might be a rather good match for $T$ Tauri and its nebulosity.

These results suggest the possibility that at least some of the stars like $\mathrm{T}$ Tauri may have begun their histories as Herbig-Haro Objects[10]. Speculations that these Objects are somehow connected with star formation have been encouraged by the recent discovery that two objects which seem to be stellar have appeared in one of the brighter Herbig-Haro Objects sometime between January 1947 and December 1954 (see Fig. 3).
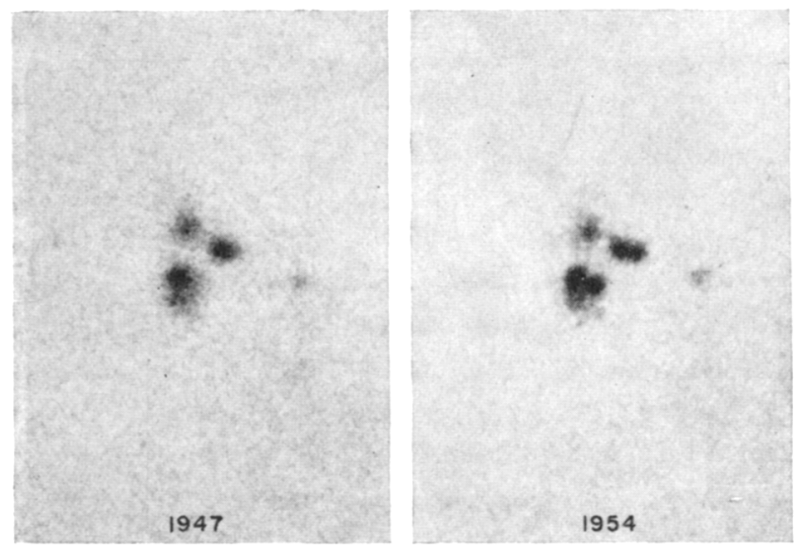

Fig. 3. Direct photographs of the Object Haro $12 a=$ Herbig 2, in Orion. The plates were obtained on 20 January 1947 and 20 December 1954 with the Crossley reflector and blue-sensitive emulsions. North is at the top and east at the right. The scale can be obtained from the fact that the two brightest stars on the 1947 photograph are 8" apart.

The $1946-7$ Lick photographs show this particular Object to have a complex structure: it is composed of a pair of stars 8 " apart of about photographic magnitude 17 , probably 3 much fainter stars, and several small masses of nebulosity, all in an area about $20^{\prime \prime}$ across. On the 1954-5 plates, two additional stars are present, each lying 3 to 4 " from a component of the original pair of 8 " separation. Photographically, these newly-appeared objects have brightened up through ranges of at least 3 magnitudes. Their photographic magnitudes are now about 17 , but much of this energy is concentrated in the emission lines. Direct photo-

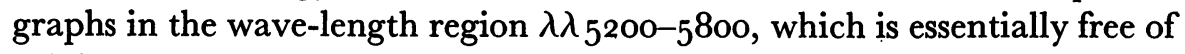
bright lines, show that in this spectral region the stars as well as the nebulosity are at least one magnitude fainter than in the photographic region. The 'visual' absolute magnitudes, with the contribution of emission lines excluded but without allowance for selective absorption, 
must therefore be about +9 at the present time. No large changes have been observed in the brightness of these newly-appeared objects during two months of the 1954-5 observing season, but one would be rash to attempt a prediction of their behaviour in the years to come.*

Our understanding of what may be taking place in this Object could hardly be more incomplete, but perhaps we have witnessed the opening phase of an episode in stellar evolution. It may be in this way that a $\mathrm{T}$ Tauri star begins its history, and I have emphasized that possibility here. But the structure of the particular Herbig-Haro Object in which these two 'new' stars have been found suggests an alternative evolutionary interpretation, as follows: We know that the $\mathrm{T}$ Tauri stars often occur in pairs, and occasionally in loose groups, but this Object now contains seven star-like objects within a very small area. The space density is about ${ }_{10}{ }^{5}$ stars per cubic parsec. Such a concentration is highly reminiscent of the complex multiple star systems often found in O-associations[15], of which a well-known example, the Trapezium of the Orion Nebula, lies $I{ }^{\circ} 5$ away. Therefore, perhaps this particular Herbig-Haro Object is near the beginning of the evolutionary road that leads upward toward the highluminosity O- or B-type multiple systems. The question of whether the $\mathrm{T}$ Tauri stars are to be identified with an intermediate stage along this road, or whether they define an entirely separate path, forms a subject for future investigation.

One way in which one might learn more of the relationship of the $\mathrm{T}$ Tauri stars to the Herbig-Haro Objects is to find stars in an intermediate state of development, under the assumption that they would be recognizable as such. A search for objects of this type has been underway at the Lick Observatory for some time, and one promising prospect has been found.

(6) It is now possible to make an additional point regarding the nature of the T Tauri stars, which it was necessary to postpone until now. The Lick direct photographs show that the newly-appeared objects in Orion certainly did not develop out of any stars that had been visible at those positions before. If these 'new' stars are to be identified with the beginning of the development of a T Tauri star, then this evidence (within its limitations as to absolute magnitude) also supports the hypothesis that the $\mathrm{T}$ Tauri stars are not rejuvenated interlopers from the surrounding field, but are, in fact, newly-formed objects. The same conclusion is to be drawn, of course, if the newly-appeared objects are the beginnings of $\mathrm{O}$ - or B-type stars.

\footnotetext{
* Plates taken in September and December 1955 showed that no further change had taken place in the appearance of this Object since the observations of December 1954-February 1955.
} 


\section{REFERENGES}

[1] G. H. Herbig, Four. R.A.S. Canada, 46, 222 (1952).

[2] G. H. Herbig and J. F. Spalding, Jr, Ap.J. 121, I 8 (1955); Contr. Lick Obs. Ser. II, No. 56 .

[3] M. F. Walker, Sky and Telescope, 13, 426 (1954); Ap. F. Suppl. 2 (No. 23), 365 (1956).

[4] A. H. Joy, Ap.J. 102, I68 (1945); 110, 424 (1949).

[5] R. F. Sanford, Publ. A.S.P. 59, I 34 (1947); G. H. Herbig, unpublished.

[6] E. E. Salpeter, Mem. Soc. Roy. Sci. Liège, quatr. série, 14, I 6 (1954).

[7] L. G. Henyey et al., Publ. A.S.P. 67, I54 (1955).

[8] G. Haro, B. Iriarte and E. Chavira, Bol. Obs. Tonantzintla y Tacubaya, No. 8, 8 (1953).

[9] G. H. Herbig, unpublished.

[10] V. Ambartsumian, Comm. Burakan Obs. No. 13 (1954).

[I I] G. H. Herbig, Ap.F. 113, 697 (1951).

[12] G. Haro, $A p . \mathcal{F} .115,572$ (1952); 117, 73 (1953).

[13] K.-H. Böhm, Publ. A.S.P. 67, $33^{8}$ (1955); Ap. F. 123, 379 (1956).

[14] G. H. Herbig, $A p .7 .111$, i (1949).

[15] S. Sharpless, Ap.J. 119, 334 (1954). 